\title{
BIFURCATION OF SOLUTIONS OF THE SECOND ORDER BOUNDARY VALUE PROBLEMS IN HILBERT SPACES
}

\author{
A.A. BOICHUK AND O.O. POKUTNYI
}

Received 20 February, 2019

\begin{abstract}
Bifurcations conditions of solutions of perturbed linear boundary value problems in the Hilbert spaces for the second order evolution equation are obtained.

2010 Mathematics Subject Classification: 35L20; 34K18; 34K10

Keywords: bifurcation, resonance case, Moore-Penrose pseudoinverse operator, generalized solution, hyperbolic equation
\end{abstract}

\section{INTRODUCTION}

It is known that differential equations of the second order in Hilbert spaces play important role in the qualitative theory of differential equations, probability theory and stochastic processes. It should be noted some books and papers in this direction [7] - [1].

As a rule such problems were investigated in the regular case when the corresponding linear problem has unique solution. In the presented paper we consider boundary value problem for the second order evolution equation in Hilbert spaces (resonance case). We obtain the necessary and sufficient conditions of the existence of solutions of such problem in the critical (irregular) case when considering linear problem can have set of solutions not for any right hand sides. Bifurcation conditions of solutions are obtained in general case.

\section{STATEMENT OF THE PROBLEM}

Consider the following boundary value problem (BVP)

$$
\begin{gathered}
y^{\prime \prime}(t, \varepsilon)+A(t) y(t, \varepsilon)=\varepsilon A_{1}(t) y(t, \varepsilon)+f(t), \\
l\left(y(\cdot, \varepsilon), y^{\prime}(\cdot, \varepsilon)\right)^{T}=\alpha,
\end{gathered}
$$

where $y: J \rightarrow \mathscr{H}$ is a vector-function $y \in C^{2}(J, \mathscr{H})$ with values in a Hilbert space $\mathscr{H}$, $J \subset R$, the closed operator-valued function $A(t)$ acts from $J$ into the dense domain $D=D(A(t)) \subset \mathscr{H}$ which is independent from $t, l$ is a linear and bounded operator 
which translates solutions of (2.1) into the Hilbert space $\mathscr{H}_{1}, A_{1}(t)$ is a linear and bounded operator valued function $\left\|\left|A_{1}\right|\right\|=\sup _{t \in J}\left\|A_{1}(t)\right\|<\infty, \alpha \in \mathscr{H}_{1}$. In such a way we consider the case of the so-called abstract hyperbolic equation.

\section{LINEAR CASE}

At first we find the necessary and sufficient conditions of the existence of solutions of linear unperturbed nonhomogeneous boundary value problem

$$
y_{0}^{\prime \prime}(t)+A(t) y_{0}(t)=f(t), \quad l\left(y_{0}(\cdot), y_{0}^{\prime}(\cdot)\right)^{T}=\alpha .
$$

Let $x_{1}^{0}(t)=y_{0}(t), x_{2}^{0}(t)=y_{0}^{\prime}(t), x_{0}(t)=\left(x_{1}^{0}(t), x_{2}^{0}(t)\right)^{T}$, then we can rewrite boundary value problem (3.1) in the form of the operator system

$$
x_{0}^{\prime}(t)=B(t) x_{0}(t)+g(t), \quad l x_{0}(\cdot)=\alpha,
$$

where

$$
B(t)=\left(\begin{array}{cc}
0 & I \\
-A(t) & 0
\end{array}\right), g(t)=(0, f(t))^{T} .
$$

Denote by $U(t)$ the evolution operator of homogeneous equation:

$$
U^{\prime}(t)=B(t) U(t), U(0)=I .
$$

Then the set of solutions of (3.2) has the form

$$
x_{0}(t, c)=U(t) c+\int_{0}^{t} U(t) U^{-1}(\tau) g(\tau) d \tau .
$$

Substituting in boundary condition $l x_{0}(\cdot)=\alpha$ we obtain the following operator equation

$$
Q c=\alpha-l \int_{0}^{\cdot} U(\cdot) U^{-1}(\tau) g(\tau) d \tau, \quad Q=l U(\cdot): \mathscr{H} \rightarrow \mathscr{H}_{1},
$$

or in the form

$$
Q c=g_{1}, c \in \mathscr{H}
$$

where $Q=l U(\cdot, 0), g_{1}=\alpha-l \int_{0}^{*} U(\cdot, \tau) f(\tau) d \tau$.

We have three types of solutions of the equation (3.5).

1) Classical generalized solutions.

Consider the case when the set of values of $Q$ is closed $(R(Q)=\overline{R(Q)})$. Then operator equation (3.5) is solvable if and only if the element $g_{1} \in R(Q)$ or $\mathcal{P}_{N\left(Q^{*}\right)} g_{1}=$ 0 [6]. Here $\mathcal{P}_{N\left(Q^{*}\right)}$ is the orthoprojector onto the cokernel of the operator $Q$. The set of solutions of (3.5) has the form

$$
c=Q^{+} g_{1}+\mathcal{P}_{N(Q)} \bar{c}, \quad \forall \bar{c} \in \mathcal{H} .
$$

2) Strong generalized solutions.

Consider the case where $R(Q) \neq \overline{R(Q)}$ and $g_{1} \in \overline{R(Q)}$. We show that the operator $Q$ can be extended to the operator $\bar{Q}$ in such a way that $R(\bar{Q})$ is closed. It gives 
possibility to define the notion of strong generalized solutions and obtain solvability condition of the corresponding equation in the case $R(Q) \neq \overline{R(Q)}$.

Since the operator $Q$ is linear and bounded, spaces $\mathscr{H}$ and $\mathscr{H}_{1}$ can be represented in the form of a direct sums of subspaces:

$$
\mathscr{H}=N(Q) \oplus X, \quad \mathscr{H}_{1}=\overline{R(Q)} \oplus Y,
$$

where $X=N(Q)^{\perp}, Y=\overline{R(Q)}^{\perp}$. Let $\mathscr{H}_{2}=\mathscr{H} / N(Q)$ be the quotient space of $\mathscr{H}$ and let $\mathcal{P}_{\overline{R(Q)}}$ and $\mathcal{P}_{N(Q)}$ be the orthoprojectors onto $\overline{R(Q)}$ and $N(Q)$, respectively. Then the operator

$$
Q=\mathcal{P}_{\overline{R(Q)}} Q j^{-1} p: X \rightarrow R(Q) \subset \overline{R(Q)}
$$

is linear, continuous, and injective. Here

$$
p: X \rightarrow \mathscr{H}_{2}, j: \mathscr{H} \rightarrow \mathscr{H}_{2}
$$

are continuous bijection and projection, respectively [2]. The triple $\left(\mathscr{H}_{,} \mathscr{H}_{2}, j\right)$ is a locally trivial bundle with a typical fiber $\mathcal{P}_{N(Q)} \mathscr{H}$. In this case, we can define a strong generalized solution of the equation

$$
Q c=g_{1}, c \in X \text {. }
$$

We complete the space $X$ by the norm $\|x\|_{\bar{X}}=\|\mathcal{Q} x\|_{F}$ [8], where $F=\overline{R(Q)}$. Then the extended operator

$$
\bar{Q}: \bar{X} \rightarrow R(Q) \subset \overline{R(Q)}
$$

is a homeomorphism of $\bar{X}$ and $\overline{R(Q)}$. By the construction of a strong generalized solution, the equation

$$
\bar{Q} \bar{c}=g_{1},
$$

has a unique solution $\overline{\mathcal{Q}}^{-1} g_{1}$, which is called the strong generalized solution of equation (3.6).

In such a way we obtain an operator $\bar{Q}=\overline{\mathcal{Q}} \mathcal{P}_{\bar{X}}: \overline{\mathscr{H}} \rightarrow \mathscr{H}_{1}$ which is normally resolvable $(R(\bar{Q})=\overline{R(\bar{Q})})$ and has Moore-Penrose pseudoinverse $\bar{Q}^{+}, \overline{\mathscr{H}}=N(Q) \oplus$ $\bar{X}$. Then the set of strong generalized solutions of the equation (3.5) has the form [6]:

$$
c=\bar{Q}^{+} g_{1}+\mathcal{P}_{N(\bar{Q})} \bar{c}, \forall \bar{c} \in \overline{\mathscr{H}} .
$$

3) Strong pseudosolutions.

Consider the case when element $g_{1} \notin \overline{R(Q)} \neq R(Q)$. This condition is equivalent to the following $\mathcal{P}_{N\left(Q^{*}\right)} g_{1} \neq 0$. In this case, there are elements $c$ of $\overline{\mathscr{H}}$ that minimize the norm $\left\|\bar{Q} c-g_{1}\right\|_{\mathscr{H}}$ :

$$
c=\bar{Q}^{+} g_{1}+\mathcal{P}_{N(\bar{Q})} \bar{c}, \bar{c} \in \overline{\mathscr{H}} .
$$

These elements are called strong pseudosolutions by analogy with [6], [8].

Considered cases give possibility to formulate the following result. 
Theorem 1. 1. a) Boundary value problem (3.1) has strong generalized solutions if and only if the following condition holds

$$
\mathcal{P}_{N\left(\bar{Q}^{*}\right)}\left\{\alpha-l \int_{0}^{\cdot} U(\cdot) U^{-1}(\tau) g(\tau) d \tau\right\}=0
$$

if

$$
\alpha-l \int_{0}^{\cdot} U(\cdot) U^{-1}(\tau) g(\tau) d \tau \in R(Q),
$$

then strong generalized solutions are classical;

b) under condition (3.7) the set of solutions has the form

$$
x_{0}(t, c)=U(t) \mathcal{P}_{N(\bar{Q})} c+\overline{(G[g, \alpha])(t)}, \quad \forall c \in \mathscr{H}
$$

where $\mathcal{P}_{N(\bar{Q})}, \mathcal{P}_{N\left(\bar{Q}^{*}\right)}$ are the orthoprojectors onto the kernel and cokernel of the operator $\bar{Q}$ respectively,

$$
\overline{(G[g, \alpha])(t)}=\int_{0}^{t} U(t) U^{-1}(\tau) g(\tau) d \tau+\bar{Q}^{+}\left\{\alpha-l \int_{0}^{\cdot} U(\cdot) U^{-1}(\tau) g(\tau) d \tau\right\}
$$

is a generalized Green operator of the boundary value problem (3.1);

2. a) Boundary value problem (3.1) has strong pseudosolutions if and only if the following condition holds

$$
\mathcal{P}_{N\left(\bar{Q}^{*}\right)}\left\{\alpha-l \int_{0}^{\cdot} U(\cdot) U^{-1}(\tau) g(\tau) d \tau\right\} \neq 0
$$

b) under condition (3.8) the set of strong pseudosolutions has the form

$$
x_{0}(t, c)=U(t) \mathcal{P}_{N(\bar{Q})} c+\overline{(G[g, \alpha])(t)}, \quad \forall c \in \mathcal{H} .
$$

\section{BIFURCATION CONDITIONS}

a) Suppose that condition (3.8) is hold. Our main goal is to obtain conditions for $A_{1}(t)$ which guarantee strong generalized solvability of perturbed boundary value problem

$$
\begin{gathered}
x^{\prime}(t, \varepsilon)=B(t) x(t, \varepsilon)+g(t)+\varepsilon B_{1}(t) x(t, \varepsilon), \\
l x(\cdot, \varepsilon)=\alpha .
\end{gathered}
$$

Here is an operator-valued function $B_{1}(t)$ has the following form:

$$
B_{1}(t)=\left(\begin{array}{cc}
A_{1}(t) & 0 \\
0 & A_{2}(t)
\end{array}\right), g(t)=(0, f(t))^{T},
$$

$x(t, \varepsilon)=\left(x_{1}(t, \varepsilon), x_{2}(t, \varepsilon)\right)^{T}, x_{1}(t, \varepsilon)=y(t, \varepsilon), x_{2}(t, \varepsilon)=y^{\prime}(t, \varepsilon)$. We will use the modification of the well-known Vishik-Lyusternik method [9]. A solution of problem 
(4.1), (4.2) is sought in the form of a segment of the series in powers of the small parameter $\varepsilon$ :

$$
x(t, \varepsilon)=\sum_{i=-1}^{+\infty} \varepsilon^{i} x_{i}(t)=\frac{x_{-1}(t)}{\varepsilon}+x_{0}(t)+\varepsilon x_{1}(t)+\varepsilon^{2} x_{2}(t)+\ldots
$$

Substituting series (4.4) into problem (4.1), (4.2) and equating the coefficients of $\varepsilon^{k}$. For $\varepsilon^{-1}$ we obtain the following boundary value problem for finding the coefficient $x_{-1}(t)$ of series (4.4):

$$
\begin{gathered}
x_{-1}^{\prime}(t)=B(t) x_{-1}(t), \\
l x_{-1}(\cdot)=0 .
\end{gathered}
$$

Problem (4.5), (4.6) has a family of solutions:

$$
x_{-1}\left(t, c_{-1}\right)=U(t) \mathcal{P}_{N(\bar{Q})} c_{-1}, \quad \forall c_{-1} \in \mathcal{H} .
$$

An arbitrary element $c_{-1}$ is determined by the condition for the solvability of the following linear inhomogeneous boundary value problem for finding the coefficient $x_{0}(t)$ in series (4.4):

$$
\begin{gathered}
x_{0}^{\prime}(t)=B(t) x_{0}(t)+B_{1}(t) x_{-1}(t)+g(t), \\
l x_{0}(\cdot)=\alpha .
\end{gathered}
$$

A necessary and sufficient condition for the solvability of the problem (4.7), (4.8) is given by

$$
\mathcal{P}_{N\left(\bar{Q}^{*}\right)}\left\{\alpha-l \int_{0}^{\cdot} U(\cdot) U^{-1}(\tau)\left(B_{1}(\tau) x_{-1}\left(\tau, c_{-1}\right)+g(\tau)\right) d \tau\right\}=0 .
$$

From this, in view of the form of $x_{-1}\left(t, c_{-1}\right)$, we obtain an operator equation for $c_{-1} \in \mathscr{H}$ :

$$
B_{0} c_{-1}=\mathcal{P}_{N\left(\bar{Q}^{*}\right)}\left\{\alpha-l \int_{0}^{\cdot} U(\cdot) U^{-1}(\tau) g(\tau) d \tau\right\}
$$

where

$$
B_{0}=\mathcal{P}_{N\left(\bar{Q}^{*}\right)} l \int_{0}^{\cdot} U(\cdot) U^{-1}(\tau) B_{1}(\tau) U(\tau) d \tau \mathcal{P}_{N(\bar{Q})} .
$$

A necessary and sufficient condition of generalized solvability has the following form

$$
\mathcal{P}_{N\left(\bar{B}_{0}^{*}\right)} \mathcal{P}_{N\left(\bar{Q}^{*}\right)}\left\{\alpha-l \int_{0}^{\cdot} U(\cdot) U^{-1}(\tau) g(\tau) d \tau\right\}=0
$$

Here $\mathcal{P}_{N\left(\bar{B}_{0}^{*}\right)}, \mathcal{P}_{N\left(\bar{Q}^{*}\right)}$ are orthoprojectors onto cokernels of extended adjoint operators $\bar{B}_{0}^{*}, \bar{Q}^{*}$ respectively. Suppose that $\mathcal{P}_{N\left(\bar{B}_{0}^{*}\right)} \mathcal{P}_{N\left(\bar{Q}^{*}\right)}=0$. Then condition (4.10) is hold. The solution set of operator equation for $c_{-1} \in \mathscr{H}$ has the form

$$
c_{-1}=\bar{c}_{-1}+\mathcal{P}_{N\left(\bar{B}_{0}\right)} c_{\rho}, \quad \forall c_{\rho} \in \mathcal{H},
$$


where

$$
\bar{c}_{-1}=\bar{B}_{0}^{+} \mathcal{P}_{N\left(\bar{Q}^{*}\right)}\left\{\alpha-l \int_{0}^{\cdot} U(\cdot) U^{-1}(\tau) g(\tau) d \tau\right\} .
$$

In view of the expression for $c_{-1}$, the homogeneous boundary value problem (4.5), (4.6) has a $\rho$ - parameter family of solutions

$$
x_{-1}\left(t, c_{\rho}\right)=\bar{x}_{-1}\left(t, \bar{c}_{-1}\right)+U(t) \mathcal{P}_{N(\bar{Q})} \mathcal{P}_{N\left(\bar{B}_{0}\right)} c_{\rho},
$$

where

$$
\bar{x}_{-1}\left(t, \bar{c}_{-1}\right)=U(t) \mathcal{P}_{N(\bar{Q})} \bar{c}_{-1} .
$$

The general solution of the problem (4.7), (4.8) has the form

$$
x_{0}\left(t, c_{0}\right)=U(t) \mathcal{P}_{N(\bar{Q})} c_{0}+F_{-1}(t)+K_{-1}(t) \mathcal{P}_{N\left(\bar{B}_{0}\right)} c_{\rho},
$$

where

$$
F_{-1}(t)=\overline{\left(G\left[g+B_{1} \bar{x}_{-1}, \alpha\right]\right)(t)}, K_{-1}(t)=\overline{(G[U, 0])(t)} \mathcal{P}_{N(\bar{Q})},
$$

$c_{0}$ is an element of the space $\mathscr{H}$, which is determined at the next step from the condition for the solvability of the boundary value problem for finding the coefficient $x_{1}(t)$ in series (4.4). To determine the coefficient $x_{1}(t)$ of $\varepsilon^{1}$ in series (4.4), we obtain the following boundary value problem

$$
\begin{gathered}
x_{1}^{\prime}(t)=B(t) x_{1}(t)+B_{1}(t) x_{0}\left(t, c_{0}\right), \\
l x_{1}(\cdot)=0 .
\end{gathered}
$$

Under condition of solvability

$$
\mathcal{P}_{N\left(\bar{Q}^{*}\right)} l \int_{0}^{\cdot} U(\cdot) U^{-1}(\tau) B_{1}(\tau) x_{0}\left(\tau, c_{0}\right) d \tau=0,
$$

boundary value problem (4.12), (4.13) has the set of solutions in the form

$$
x_{1}\left(t, c_{1}\right)=U(t) \mathcal{P}_{N(\bar{Q})} c_{1}+\overline{\left(G\left[B_{1} U \mathcal{P}_{N(\bar{Q})} c_{0}+F_{-1}+K_{-1}, 0\right]\right)(t)} .
$$

The condition for the solvability of the boundary value problem for the element $c_{0}$ is

$$
\begin{gathered}
B_{0} c_{0}=-\mathcal{P}_{N\left(\bar{Q}^{*}\right)} l \int_{0}^{\cdot} U(\cdot) U^{-1}(\tau) B_{1}(\tau) F_{-1}(\tau) d \tau- \\
-\mathcal{P}_{N\left(\bar{Q}^{*}\right)} l \int_{0}^{\cdot} U(\cdot) U^{-1}(\tau) B_{1}(\tau) K_{-1}(\tau) d \tau \mathcal{P}_{N(\bar{Q})} \mathcal{P}_{N\left(\bar{B}_{0}\right)} c_{\rho} .
\end{gathered}
$$

From the condition $\mathcal{P}_{N\left(B_{0}^{*}\right)} \mathcal{P}_{N\left(\bar{Q}^{*}\right)}=0$ follows solvability of the equation (4.14) with the set of solutions in the following form

$$
\begin{gathered}
c_{0}=-\bar{B}_{0}^{+} \mathcal{P}_{N\left(\bar{Q}^{*}\right)} l \int_{0}^{\cdot} U(\cdot) U^{-1}(\tau) B_{1}(\tau) F_{-1}(\tau) d \tau- \\
-\bar{B}_{0}^{+} \mathcal{P}_{N\left(\bar{Q}^{*}\right)} l \int_{0}^{\cdot} U(\cdot) U^{-1}(\tau) B_{1}(\tau) K_{-1}(\tau) d \tau \mathcal{P}_{N(\bar{Q})} \mathcal{P}_{N\left(\bar{B}_{0}\right)} c_{\rho}+\mathcal{P}_{N\left(\bar{B}_{0}\right)} c_{\rho}, \\
c_{0}=\bar{c}_{0}+D_{0} \mathcal{P}_{N\left(\bar{B}_{0}\right)} c_{\rho}, \quad \forall c_{\rho} \in \mathcal{H},
\end{gathered}
$$


where

$$
\begin{gathered}
\bar{c}_{0}=-\bar{B}_{0}^{+} \mathcal{P}_{N\left(\bar{Q}^{*}\right)} l \int_{0}^{\cdot} U(\cdot) U^{-1}(\tau) B_{1}(\tau) F_{-1}(\tau) d \tau, \\
D_{0}=I-\bar{B}_{0}^{+} \mathcal{P}_{N\left(\bar{Q}^{*}\right)} l \int_{0}^{\cdot} U(\cdot) U^{-1}(\tau) B_{1}(\tau) K_{-1}(\tau) d \tau \mathcal{P}_{N(\bar{Q})} .
\end{gathered}
$$

Thus, problem (4.7), (4.8) has a $\rho$-parameter family of solutions:

$$
x_{0}\left(t, c_{0}\right)=\bar{x}_{0}\left(t, \bar{c}_{0}\right)+\bar{X}_{0}(t) \mathcal{P}_{N\left(\bar{B}_{0}\right)} c_{\rho}, \quad \forall c_{\rho} \in \mathcal{H},
$$

where

$$
\begin{gathered}
\bar{x}_{0}\left(t, \bar{c}_{0}\right)=U(t) \mathcal{P}_{N(\bar{Q})} \bar{c}_{0}+F_{-1}(t), \\
\bar{X}_{0}(t)=U(t) \mathcal{P}_{N(\bar{Q})} D_{0}+K_{-1}(t) .
\end{gathered}
$$

Then problem (4.12), (4.13) has a $\rho$ - parameter family of solutions

$$
x_{1}\left(t, c_{1}\right)=U(t) \mathcal{P}_{N(\bar{Q})} c_{1}+F_{0}(t)+K_{0}(t) \mathcal{P}_{N\left(B_{0}\right)} c_{\rho},
$$

where

$$
\begin{gathered}
F_{0}(t)=\overline{\left(G\left[B_{1} U \mathcal{P}_{N(\bar{Q})} \bar{c}_{0}+F_{-1}+K_{-1}, 0\right]\right)(t)}, \\
K_{0}(t)=\overline{\left(G\left[B_{1} U \mathcal{P}_{N(\bar{Q})} D_{0}, 0\right]\right)(t)},
\end{gathered}
$$

$c_{1}$ is an element of the Hilbert space $\mathscr{H}$, which is determined at the next step from the condition for the solvability of the boundary value problem for finding the coefficient $x_{2}(t)$ in series (4.4). By induction, we can prove that the coefficients $x_{i}(t)$ in series (4.4) are determined by solving the boundary value problem

$$
\begin{gathered}
x_{i}^{\prime}(t)=B(t) x_{i}(t)+B_{1}(t) x_{i-1}\left(t, c_{i-1}\right), \\
l x_{i}(\cdot)=0,
\end{gathered}
$$

which under the same condition of solvability $\mathcal{P}_{N\left(B_{0}^{*}\right)} \mathcal{P}_{N\left(\bar{Q}^{*}\right)}=0$ has a $\rho$-parameter family of solutions

$$
x_{i}\left(t, c_{i}\right)=\bar{x}_{i}\left(t, \bar{c}_{i}\right)+\bar{X}_{i}(t) \mathcal{P}_{N\left(\bar{B}_{0}\right)} c_{\rho}, \quad \forall c_{\rho} \in \mathcal{H}
$$

where all the terms are determined by the iterative procedure

$$
\begin{gathered}
\bar{x}_{i}\left(t, \bar{c}_{i}\right)=U(t) \mathcal{P}_{N(\bar{Q})} \bar{c}_{i}+F_{i-1}(t), \\
\bar{X}_{i}(t)=U(t) \mathcal{P}_{N(\bar{Q})} D_{i}+K_{i-1}(t), \\
D_{i}=I-\bar{B}_{0}^{+} \mathcal{P}_{N\left(\bar{Q}^{*}\right)} l \int_{0}^{\cdot} U(\cdot) U^{-1}(\tau) B_{1}(\tau) K_{i-1}(\tau) d \tau \mathcal{P}_{N(\bar{Q})}, \\
F_{i-1}(t)=\overline{\left(G\left[B_{1} U \mathcal{P}_{N(\bar{Q})} \bar{c}_{i-1}+F_{i-2}+K_{i-2}, 0\right]\right)(t)} \\
K_{i-1}(t)=\overline{\left(G\left[B_{1} U \mathcal{P}_{N(\bar{Q})} D_{i-1}, 0\right]\right)(t)} .
\end{gathered}
$$

The convergence of series (4.4) for sufficiently small fixed $\varepsilon \in\left(0, \varepsilon^{*}\right]$ is proved in the same manner as in [3]. Thus, the following result for perturbed boundary value problem (4.1), (4.2) holds. 
Theorem 2. Suppose that for unperturbed boundary value problem (3.2) condition (3.8) is hold. Under condition

$$
\mathcal{P}_{N\left(\bar{B}_{0}^{*}\right)} \mathcal{P}_{N\left(\bar{Q}^{*}\right)}=0
$$

the perturbed boundary value problem (4.1), (4.2) has a $\rho$-parameter family of strong generalized solutions in the form of the convergent series segment

$$
x\left(t, c_{\rho}\right)=\sum_{i=-1}^{+\infty} \varepsilon^{i}\left[\bar{x}_{i}\left(t, \bar{c}_{i}\right)+\bar{X}_{i}(t) \mathcal{P}_{N\left(\bar{B}_{0}\right)} c_{\rho}\right], \quad \forall c_{\rho} \in \mathcal{H},
$$

whose coefficients are given by formulas (4.18)-(4.22).

b) Suppose that condition (3.7) is hold. We obtain the condition on $A_{1}(t)$ such that the perturbed boundary value problem

$$
\begin{gathered}
x^{\prime}(t, \varepsilon)=B(t) x(t, \varepsilon)+g(t)+\varepsilon B_{1}(t) x(t, \varepsilon), \\
l x(\cdot, \varepsilon)=\alpha,
\end{gathered}
$$

has strong generalized solutions. As in previous case a solution of problem (4.24), (4.25) is sought in the form of a segment of the series in powers of the small parameter $\varepsilon$ :

$$
x(t, \varepsilon)=\sum_{i=0}^{+\infty} \varepsilon^{i} x_{i}(t)=x_{0}(t)+\varepsilon x_{1}(t)+\varepsilon^{2} x_{2}(t)+\ldots .
$$

Substituting series (4.26) into problem (4.24), (4.25) and equating the coefficients of $\varepsilon^{k}$. For $\varepsilon^{0}$, we obtain the following boundary value problem for finding the coefficient $x_{0}(t)$ of series (4.26):

$$
\begin{gathered}
x_{0}^{\prime}(t)=B(t) x_{0}(t)+g(t), \\
l x_{0}(\cdot)=\alpha .
\end{gathered}
$$

Problem (4.27), (4.28) has a family of strong generalized solutions:

$$
x_{0}\left(t, c_{0}\right)=U(t) \mathcal{P}_{N(\bar{Q})} c_{0}+\overline{(G[g, \alpha])(t)} .
$$

An arbitrary element $c_{0} \in \mathscr{H}$ is determined by the condition for the solvability of the following linear inhomogeneous boundary value problem for finding the coefficient $x_{1}(t)$ in series (4.26):

$$
\begin{gathered}
x_{1}^{\prime}(t)=B(t) x_{1}(t)+B_{1}(t) x_{0}\left(t, c_{0}\right), \\
l x_{1}(\cdot)=0 .
\end{gathered}
$$

A necessary and sufficient condition for the solvability of problem (4.29), (4.30) is given by

$$
\mathcal{P}_{N\left(\bar{Q}^{*}\right)}\left\{l \int_{0}^{\cdot} U(\cdot) U^{-1}(\tau) B_{1}(\tau) x_{0}\left(\tau, c_{0}\right) d \tau\right\}=0 .
$$


From this, in view of the form of $x_{0}\left(t, c_{0}\right)$, we obtain an operator equation for $c_{0} \in \mathscr{H}$ :

$$
B_{0} c_{0}=-\mathcal{P}_{N\left(\bar{Q}^{*}\right)} l \int_{0}^{\cdot} U(\cdot) U^{-1}(\tau) B_{1}(\tau) \overline{(G[g, \alpha])(\tau)} d \tau .
$$

Under condition $\mathcal{P}_{N\left(\bar{B}_{0}^{*}\right)} \mathcal{P}_{N\left(\bar{Q}^{*}\right)}=0$ the equation (4.31) is solvable. The solution set of operator equation for $c_{0} \in \mathscr{H}$ has the form

$$
c_{0}=\bar{c}_{0}+\mathcal{P}_{N\left(\bar{B}_{0}\right)} c_{\rho}, \forall c_{\rho} \in \mathscr{H},
$$

where

$$
\bar{c}_{0}=-\bar{B}_{0}^{+} \mathcal{P}_{N\left(\bar{Q}^{*}\right)} l \int_{0}^{\cdot} U(\cdot) U^{-1}(\tau) B_{1}(\tau) \overline{(G[g, \alpha])(\tau)} d \tau .
$$

In view of the expression for $c_{0}$, the homogeneous boundary value problem (4.27), (4.28) has a $\rho$ - parameter family of solutions

$$
x_{0}\left(t, c_{\rho}\right)=\bar{x}_{0}\left(t, \bar{c}_{0}\right)+U(t) \mathcal{P}_{N(\bar{Q})} \mathcal{P}_{N\left(\bar{B}_{0}\right)} c_{\rho}
$$

where

$$
\bar{x}_{0}\left(t, \bar{c}_{0}\right)=U(t) \mathcal{P}_{N(\bar{Q})} \bar{c}_{0}+\overline{(G[g, \alpha])(t)} .
$$

The general solution of problem (4.29), (4.30) has the form

$$
x_{1}\left(t, c_{1}\right)=U(t) \mathcal{P}_{N(\bar{Q})} c_{1}+F_{0}(t)+K_{0}(t) \mathcal{P}_{N\left(\bar{B}_{0}\right)} c_{\rho},
$$

where

$$
F_{0}(t)=\overline{\left(G\left[B_{1} \bar{x}_{0}, 0\right]\right)(t)}, K_{0}(t)=\overline{\left(G\left[B_{1} U \mathcal{P}_{N(\bar{Q})}, 0\right]\right)(t)},
$$

$c_{1}$ is an element of the space $\mathscr{H}$, which is determined at the next step from the condition for the solvability of the boundary value problem for finding the coefficient $x_{2}(t)$ in series (4.26). By induction, we can prove that the coefficients $x_{i}(t)$ in series (4.26) are determined by solving the boundary value problem

$$
\begin{gathered}
x_{i}^{\prime}(t)=B(t) x_{i}(t)+B_{1}(t) x_{i-1}\left(t, c_{i-1}\right), \\
l x_{i}(\cdot)=0,
\end{gathered}
$$

which under condition of solvability has a $\rho$-parameter family of solutions

$$
x_{i}\left(t, c_{i}\right)=\bar{x}_{i}\left(t, \bar{c}_{i}\right)+\bar{X}_{i}(t) \mathcal{P}_{N\left(\bar{B}_{0}\right)} c_{\rho}, \quad \forall c_{\rho} \in \mathcal{H}
$$

where all the terms are determined (as in previous case) by the iterative procedure (4.18)-(4.22).

The convergence of series (4.26) is proved in the same manner as in [3]. Thus, the following result holds.

Theorem 3. Suppose that for unperturbed boundary value problem (3.2) condition (3.7) is hold. Under condition

$$
\mathcal{P}_{N\left(\bar{B}_{0}^{*}\right)} \mathcal{P}_{N\left(\bar{Q}^{*}\right)}=0
$$


the boundary value problem (4.24), (4.25) has a $\rho$-parameter family of strong generalized solutions in the form of the convergent series segment

$$
x\left(t, c_{\rho}\right)=\sum_{i=0}^{+\infty}\left[\bar{x}_{i}\left(t, \bar{c}_{i}\right)+\bar{X}_{i}(t) \mathcal{P}_{N\left(\bar{B}_{0}\right)} c_{\rho}\right], \quad \forall c_{\rho} \in \mathcal{H},
$$

whose coefficients are given by formulas (4.18)-(4.22).

Remark 1 . In the case when $g_{1} \in R(Q)=\overline{R(Q)}$ under the same condition we can obtain classical generalized solutions for the system (4.24), (4.25).

Remark 2. Proposed theory gives possibility to investigate branching of solutions of boundary value problem (3.1) (see [5], [4]).

\section{EXAMPLE}

Now we find bifurcation conditions of solutions of periodic boundary value problem for hyperbolic equation in a separable (for simplicity) Hilbert space $\mathscr{H}$ :

$$
\begin{gathered}
y^{\prime \prime}(t, \varepsilon)+T y(t, \varepsilon)=\varepsilon A_{1}(t) y(t, \varepsilon)+f(t), \\
y(0, \varepsilon)=y(w, \varepsilon), y^{\prime}(0, \varepsilon)=y^{\prime}(w, \varepsilon),
\end{gathered}
$$

where $T$ is an unbounded operator with compact inverse $T^{-1}$. Suppose for example that the operator $A_{1}(t)$ is diagonal: $A_{1}(t)=\operatorname{diag}\left\{a_{i i}(t)\right\}_{i \in N}$. Then there is orthonormal basis $e_{i} \in \mathscr{H}$ such that $y(t)=\sum_{i=1}^{\infty} c_{i}(t) e_{i}, T y(t)=\sum_{i=1}^{\infty} \lambda_{i} c_{i}(t) e_{i}, \lambda_{i} \rightarrow$ $\infty$. In that case boundary value problem (5.1), (5.2) is equivalent to the following countable system of ordinary differential equations:

$$
\begin{gathered}
x_{k}^{\prime}(t, \varepsilon)=\sqrt{\lambda_{k}} y_{k}(t, \varepsilon), \\
y_{k}^{\prime}(t, \varepsilon)=-\sqrt{\lambda_{k}} x_{k}(t, \varepsilon)+\frac{1}{\sqrt{\lambda_{k}}}\left(\varepsilon a_{k k}(t) x_{k}(t, \varepsilon)+f_{k}(t)\right), \\
x_{k}(0, \varepsilon)=x_{k}(w, \varepsilon), \quad y_{k}(0, \varepsilon)=y_{k}(w, \varepsilon),
\end{gathered}
$$

where $c_{k}(t)=x_{k}(t), x_{k}^{\prime}(t)=y_{k}(t)$. Consider critical case, when $\lambda_{k}=\frac{4 \pi^{2} k^{2}}{w^{2}}$. Suppose that $w=2 \pi$. Then $\lambda_{k}=k^{2}$. At first we find conditions of solvability of generating $(\varepsilon=0)$ boundary value problem

$$
\begin{gathered}
x_{k}^{\prime}(t)=\sqrt{\lambda_{k}} y_{k}(t), \\
y_{k}^{\prime}(t)=-\sqrt{\lambda_{k}} x_{k}(t)+\frac{1}{\sqrt{\lambda_{k}}} f_{k}(t), \\
x_{k}(0)=x_{k}(w), \quad y_{k}(0)=y_{k}(w),
\end{gathered}
$$

It is easy to see, that generating boundary value problem (5.5), (5.6) is solvable if and only if

$$
\int_{0}^{2 \pi} \sin (k \tau) f_{k}(\tau) d \tau=0
$$




$$
\int_{0}^{2 \pi} \cos (k \tau) f_{k}(\tau) d \tau=0, k \in N
$$

Under condition (5.7), (5.8) generating boundary value problem (5.5), (5.6) has the set of periodic solutions in the form

$$
\left(\begin{array}{c}
x_{k}\left(t, c_{1}^{k}, c_{2}^{k}\right) \\
y_{k}\left(t, c_{1}^{k}, c_{2}^{k}\right)
\end{array}\right)=\left(\begin{array}{cc}
\cos k t & \sin k t \\
-\sin k t & \cos k t
\end{array}\right)\left(\begin{array}{c}
c_{1}^{k} \\
c_{2}^{k}
\end{array}\right)+\frac{1}{k}\left(\begin{array}{c}
\int_{0}^{t} \sin k(t-\tau) f_{k}(\tau) d \tau \\
\int_{0}^{t} \cos k(t-\tau) f_{k}(\tau) d \tau
\end{array}\right) .
$$

Suppose for example that $a_{k k}(t)=a_{k k} \neq 0$ and

$$
\begin{gathered}
\int_{0}^{2 \pi} \sin (k \tau) f_{k}(\tau) d \tau \neq 0, \\
\int_{0}^{2 \pi} \cos (k \tau) f_{k}(\tau) d \tau \neq 0, k \in N .
\end{gathered}
$$

It means that generating boundary value problem hasn't solutions. Strong generalized solution of the problem (5.3), (5.4) we find in the form of series by the power of small parameter $\varepsilon$ :

$$
x(t, \varepsilon)=\frac{x_{-1}(t)}{\varepsilon}+x_{0}(t)+\varepsilon x_{1}(t)+\ldots
$$

Under $\varepsilon^{-1}$ we obtain the following boundary value problem for finding of coefficient $x_{-1}(t)=\left(x_{-1}^{k}(t), y_{-1}^{k}(t)\right)_{k \in N}^{T}$. For $\varepsilon^{0}$ we obtain:

$$
\begin{aligned}
& \frac{d x_{-1}^{k}(t)}{d t}=k y_{-1}^{k}(t), \quad x_{-1}^{k}(0)=x_{-1}^{k}(2 \pi), \\
& \frac{d y_{-1}^{k}(t)}{d t}=-k x_{-1}^{k}(t), \quad y_{-1}^{k}(0)=y_{-1}^{k}(2 \pi) .
\end{aligned}
$$

The set of solutions of such problem has the following form

$$
\begin{gathered}
x_{-1}^{k}\left(t, c_{-1}\right)=\cos k t c_{-1}^{1 k}+\sin k t c_{-1}^{2 k}, \\
y_{-1}^{k}\left(t, c_{-1}\right)=-\sin k t c_{-1}^{1 k}+\cos k t c_{-1}^{2 k} .
\end{gathered}
$$

Any element $c_{-1}=\left(c_{-1}^{1 k}, c_{-1}^{2 k}\right)_{k \in N}^{T}$ we can find from the condition of solvability of the following boundary value problem for the coefficient $x_{0}(t)=\left(x_{0}^{k}(t), y_{0}^{k}(t)\right)_{k \in N}^{T}$ :

$$
\begin{gathered}
\frac{d x_{0}^{k}(t)}{d t}=k y_{0}^{k}(t), x_{0}^{k}(0)=x_{0}^{k}(2 \pi), \\
\frac{d y_{0}^{k}(t)}{d t}=-k x_{0}^{k}(t)+\frac{1}{k} a_{k k} x_{-1}^{k}\left(t, c_{-1}\right)+\frac{1}{k} f_{k}(t), y_{0}^{k}(0)=y_{0}^{k}(2 \pi) .
\end{gathered}
$$

The set of solutions $x_{0}^{k}(t), y_{0}^{k}(t)$ of such problem has the following form $x_{0}^{k}\left(t, c_{0}\right)=\cos k t c_{0}^{1 k}+\sin k t c_{0}^{2 k}+\frac{1}{k} \int_{0}^{t} \sin k(t-\tau)\left(a_{k k} x_{-1}^{k}\left(\tau, c_{-1}\right)+f_{k}(\tau)\right) d \tau$, 
$y_{0}^{k}\left(t, c_{0}\right)=-\sin k t c_{0}^{1 k}+\cos k t c_{0}^{2 k}+\frac{1}{k} \int_{0}^{t} \cos k(t-\tau)\left(a_{k k} x_{-1}^{k}\left(\tau, c_{-1}\right)+f_{k}(\tau)\right) d \tau$,

if and only if the following system is solvable

$$
B_{0} c_{-1}=g \text {, }
$$

where $B_{0}$ - is a countable invertible matrix in the form of $2 \times 2$ block matrices:

$$
\begin{gathered}
B_{0}=\text { block }\left(\begin{array}{cc}
0 & -\pi a_{k k} \\
\pi a_{k k} & 0
\end{array}\right)_{k \in N}, \\
g=\left(\int_{0}^{2 \pi} \sin (k \tau) f_{k}(\tau) d \tau,-\int_{0}^{2 \pi} \cos (k \tau) f_{k}(\tau) d \tau\right)_{k \in N}^{T} .
\end{gathered}
$$

It is easy to see that condition (4.23) of the theorem 2 is hold and in this case strong generalized solution is classical solution.

Then constants $\bar{c}_{-1}^{1 k}, \bar{c}_{-1}^{2 k}$ have the form:

$$
\bar{c}_{-1}^{1 k}=\frac{-\int_{0}^{2 \pi} \cos k \tau f_{k}(\tau) d \tau}{\pi a_{k k}}, \quad \bar{c}_{-1}^{2 k}=\frac{-\int_{0}^{2 \pi} \sin k \tau f_{k}(\tau) d \tau}{\pi a_{k k}} .
$$

Finally we obtain that

$$
x_{0}^{k}\left(t, c_{0}\right)=\cos k t c_{0}^{1 k}+\sin k t c_{0}^{2 k}+\frac{1}{k} g_{0}^{1 k}(t),
$$

where

$$
\begin{gathered}
g_{0}^{1 k}(t)=\int_{0}^{2 \pi} K_{1}(t, \tau) f_{k}(\tau) d \tau+\int_{0}^{t} \sin k(t-\tau) f_{k}(\tau) d \tau, \\
K_{1}(t, \tau)=\frac{1}{2 \pi k}(k t \sin k(t-\tau)-\sin k t \sin k \tau) ; \\
y_{0}^{k}\left(t, c_{0}\right)=-\sin k t c_{0}^{1 k}+\cos k t c_{0}^{2 k}+\frac{1}{k} g_{0}^{2 k}(t),
\end{gathered}
$$

where

$$
\begin{gathered}
g_{0}^{2 k}(t)=\int_{0}^{2 \pi} K_{2}(t, \tau) f_{k}(\tau) d \tau+\int_{0}^{t} \cos k(t-\tau) f_{k}(\tau) d \tau, \\
K_{2}(t, \tau)=-\frac{1}{2 \pi k}(k t \cos k(t-\tau)+\sin k t \cos k \tau) .
\end{gathered}
$$

Any element $c_{i-1}$ we can determine from the condition of solvability of boundary value problem for the coefficient $x_{i}(t)$ under $\varepsilon^{i}$ of the series (5.11):

$$
\begin{gathered}
\frac{d x_{i}^{k}(t)}{d t}=k y_{i}^{k}(t), x_{i}^{k}(0)=x_{i}^{k}(2 \pi), \\
\frac{d y_{i}^{k}(t)}{d t}=-k x_{i}^{k}(t)+\frac{1}{k} a_{k k} x_{i-1}^{k}\left(t, c_{i-1}\right), y_{i}^{k}(0)=y_{i}^{k}(2 \pi) .
\end{gathered}
$$


The set of solutions has the form

$$
\begin{gathered}
x_{i}^{k}\left(t, c_{i}\right)=\cos k t c_{i}^{1 k}+\sin k t c_{i}^{2 k}+\frac{1}{k} g_{i}^{1 k}(t), \\
y_{i}^{k}\left(t, c_{i}\right)=-\sin k t c_{i}^{1 k}+\cos k t c_{i}^{2 k}+\frac{1}{k} g_{i}^{2 k}(t),
\end{gathered}
$$

where

$$
\begin{gathered}
g_{i}^{1 k}(t)=-a_{k k} \int_{0}^{2 \pi} K_{1}(t, \tau) g_{i-1}^{1 k}(\tau) d \tau-a_{k k} \int_{0}^{t} \sin k(t-\tau) g_{i-1}^{1 k}(\tau) d \tau \\
g_{i}^{2 k}(t)=a_{k k} \int_{0}^{2 \pi} K_{2}(t, \tau) g_{i-1}^{1 k}(\tau) d \tau+a_{k k} \int_{0}^{t} \cos k(t-\tau) g_{i-1}^{1 k}(\tau) d \tau \\
\bar{c}_{i-1}^{1 k}=-\frac{1}{\pi} \int_{0}^{2 \pi} \cos k \tau g_{i-1}^{1 k}(\tau) d \tau, \\
\bar{c}_{i-1}^{2 k}=-\frac{1}{\pi} \int_{0}^{2 \pi} \sin k \tau g_{i-1}^{1 k}(\tau) d \tau .
\end{gathered}
$$

\section{REFERENCES}

[1] N. Artamonov, "Estimates of solutions of certain classes of second-order differential equations in a hilbert space," Sb. Math., vol. 194, no. 8, pp. 1113-1123, 2003.

[2] M. Atiyah, K-theory, New-York, Amsterdam, 1967.

[3] A. Boichuk and O. Pokutnyi, "Bounded solutions of linear perturbed differential equations in a banach space," Tatra Mountains Math. Publications, vol. 38, pp. 29-41, 2007.

[4] A. Boichuk and O. Pokutnyi, "Solutions of the schrodiner equation in a hilbert space. http://www.boundaryvalueproblems.com/content/2014/1/4," Boundary value problems, 2014, doi: 10.1186/1687-2770-2014-4.

[5] A. Boichuk and O. Pokutnyi, "Perturbation theory of operator equations in the frechet and hilbert spaces," Ukrainian mathematical journal, vol. 67, pp. 1327-1335, 2016.

[6] A. Boichuk and A. Samoilenko, Generalized Inverse Operators and Fredholm Boundary-Value Problems. 2nd edition. Berlin/Boston: De Gruyter, 2016.

[7] G. Da Prato and J. Zabczyk, Second Order Partial Differential Equations in Hilbert Spaces. Cambridge University Press, 2002. doi: 10.1112/S0024609303242776.

[8] D. Klyushin, S. Lyashko, D. Nomirovskii, V. V. Semenov, and Y. I. Petunin, Generalized solutions of operator equations and extreme elements. Berlin: Springer, 212. doi: 10.1007/978-1-46140619-8.

[9] M. Vishik, "The solution of some perturbation problems for matrices and selfadjoint or nonselfadjoint differential equations i," Russian Mathematical Surveys, vol. 15, pp. 1-73, 1960.

Authors' addresses

\section{A.A. Boichuk}

Institute of mathematics of NAS of Ukraine, laboratory of boundary value problems of differential equations theory, Tereshenkivska 3, $01024 \mathrm{Kiev}$, Ukraine

E-mail address: boichuk.aa@gmail.com 


\section{O.O. Pokutnyi}

Institute of mathematics of NAS of Ukraine, laboratory of boundary value problems of differential equations theory, Tereshenkivska 3, $01024 \mathrm{Kiev}$, Ukraine

E-mail address: lenasas@gmail.com 